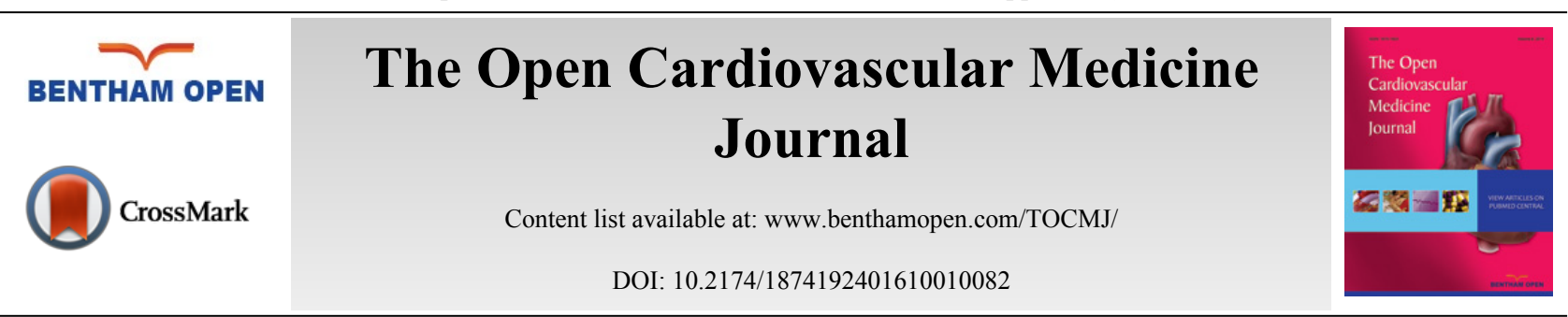

\title{
An Overview of Cardiac Management in Neuromuscular Disease
}

\author{
Renee M. Hickey, John D. Cullen and George M. Sachs* \\ Department of Neurology, Brown University, Providence, Rhode Island, 02912, USA
}

Received: September 29, 2015

Revised: October 08, 2015

Accepted: November 25, 2015

\begin{abstract}
Muscular dystrophy and myasthenia gravis are two neuromuscular disorders that can involve significant cardiovascular complications. The frequency and severity of cardiac pathology varies widely among the muscular dystrophies. In some, it is nearly inevitable and requires regular evaluation. In others, assessment of cardiac function can be more symptom-driven. On-ly a minority of myasthenic patients manifest disease-related cardiovascular complications; however, their presentation can be rapidly progressive and life-threatening.
\end{abstract}

Keywords: Arrythmia, cardiomyopathy, muscular dystrophy, myasthenia gravis, myotonic dystrophy, treatment.

\section{CARDIAC INVOLVEMENT IN MUSCULAR DYSTROPHY}

\section{Dystrophinopathies}

The dystrophinopathies comprise three types of congenital muscular dystrophy: Duchenne muscular dystrophy (DMD), Becker muscular dystrophy (BMD) and X-linked dilated cardiomyopathy. Each condition involves a mutation in the dystrophin gene which codes for a large protein that is part of the dystrophin-glycoprotein complex. There are genetic differences amongst these three muscular dystrophies and patients therefore have variable degrees of cardiac dysfunction.

\section{Duchenne Muscular Dystrophy}

Duchenne muscular dystrophy is the most common muscular dystrophy, affecting 1 in 3,500 newborn males, with a prevalence of 6/100,000 males [1]. DMD is an X-linked recessive disorder whereby mutations in the dystrophin gene disrupt the reading frame and result in absent dystrophin protein, which links cytoplasmic actin to the dystrophinglycoprotein complex at the cell membrane [2]. Muscle cell loss results in structural instability followed by necrosis of skeletal and cardiac muscle fibers. Symptomatically, boys present with weakness of leg, pelvis and shoulder girdle musculature between age 2 and 3 and are ultimately wheelchair bound by age 10 [2]. Life expectancy is usually between 15 to 25 years of age.

Cardiac involvement is common in DMD and progresses with age, with all patients over 18 exhibiting cardiac disease [1]. The process of cardiac muscle involvement is similar to that of skeletal muscle and the myocardium is eventually replaced by fibrofatty tissue. As DMD patients are typically non-ambulatory, they do not experience exercise intolerance and rather complain of sleep disturbance and loss of appetite. Cardiac disturbances include chest pain and syncope. Conduction defects and cardiomyopathy are seen after 10 years of age [1]. EKG abnormalities include tall R waves, Q waves in the lateral leads, complete/incomplete left or right bundle branch blocks and tachyarrhythmias. Echocardiography shows dilated or hypertrophic cardiomyopathy. Rhythm abnormalities include sinus arrhythmia, atrial flutter, premature atrial and ventricular beats [1]. Holter monitoring can demonstrate disordered automaticity with abnormally fast average heart rate, due to sympathetic imbalance [3]. Acute heart failure or sudden death is a cause of

\footnotetext{
* Address correspondence to this author at the Department of Neurology, Brown University, Providence, Rhode Island, 02912, USA; Tel: (401) 444-8761; Fax: (401) 444-3205; Email: gsachs@lifespan.org
} 
death in 10-20\% of DMD patients and ventricular dysfunction helps to predict mortality [1].

Cardiac evaluation in patients with DMD should occur at time of diagnosis, as $53 \%$ of children less than 5 years old will have abnormalities on EKG. Following initial evaluation, cardiac surveillance should continue every other year until symptomatic and/or yearly after age 10 [3].

\section{Becker Muscular Dystrophy}

Becker muscular dystrophy is a milder dystrophy effecting 1 in 18,450 males. Symptoms typically begin between ages 3 and 21, with a mean age of onset of 11 years old. In this X-linked recessive disease, the dystrophin gene reading frame is maintained and individuals exhibit reduced, rather than absent, levels of dystrophin [1]. BMD patients invariably have cardiac involvement, which is sometimes more pronounced than skeletal muscle weakness. Cardiomyopathy, either myocardial hypertrophy or dilated cardiomyopathy, can be a presenting feature of the disease. EKG abnormalities are similar to DMD, with tall R waves, Q waves in lateral leads, and tachyarrhthymias [1]. Death from arrhythmias or congestive heart failure occurs in up to $50 \%$ of patients, with a mean age of death of 45 years [1].

Female carriers of both DMD and BMD also require screening for cardiac disease. One study found that cardiac disease (hypertrophy, dilated cardiomyopathy or arrhythmia) develops in up to $40 \%$ of female patients [1]. As severe heart failure or sudden death may occur in female carriers, cardiac examination is recommended every 5 years.

\section{X-linked Dilated Cardiomyopathy}

$\mathrm{X}$-linked dilated cardiomyopathy is a primary myocardial dystrophinopathy. Mutations in the 5' end of the dystrophin gene lead to absence of the M-isoform in the heart and skeletal muscle (though skeletal is protected due to intact exon skipping) [1]. Preserved skeletal muscle strength increases cardiac demands, leading to cardiomyopathy [4]. Male patients typically present with congestive heart failure in their teens. Unfortunately, this is a rapidly progressive condition with death occurring within 1-2 years after incident of cardiomyopathy. Cardiac death is $100 \%$ in these males, with a mean age at death of 22 .

\section{Emery-Dreifuss Muscular Dystrophy}

There are two genetic forms of Emery-Dreifuss mucular dystrophy (EDMD) with varying cardiac involvement, Xlinked and autosomal dominant. In both forms of the disease, patients present with early contractures of the elbows, Achilles tendon and weakness in a scapulo-humeral-peroneal distribution [3].

In X-linked EDMD, mutations are seen in the emerin gene, which produces a protein component of the inner nuclear membrane. Emerin links filamentous actin to the nuclear lamina, and is absent in X-linked EDMD [1]. Prevalence of this condition is 1 in 100,000 males. Cardiac atrioventricular conduction defects are common and include bradyarrhtyhmias and tachyarrhythmias [5]. Dilated cardiomyopathy is rare. Pacemaker placement is indicated for patients with symptomatic bradycardia with heart block or atrial paralysis, secondary to atrial scarring [3]. Yearly screening with EKG and 24 hour Holter monitor is recommended, and an echocardiogram should be obtained at time of diagnosis and 5 years [5].

\section{Laminopathies}

Autosomal Dominant Emery-Dreifuss muscular dystrophy (EDMD2) is caused by a mutation in lamin A/C gene. Lamin A and $\mathrm{C}$ are proteins which provide structure to the nuclear membrane. Typically, left ventricular involvement is common with increase in age. Cardiac problems can exist even with mild skeletal muscle weakness [2].

\section{Limb Girdle Muscular Dystrophy}

Limb girdle muscular dystrophies (LGMD) are a genetically heterogeneous group of disorders. Patients present with proximal weakness between childhood and the fourth decade and the disease progresses at variable rates [2]. Prevalence amongst all forms of LGMD ranges from 1 in 23,000 to 1 in 150,000 [1]. Cardiac abnormalities vary among the subtypes and LGMD1B has clinically significant cardiac findings.

LGMD1B is a laminopathy with a similar phenotype to EDMD2, however patients present with pelvic girdle weakness later in life [1]. Dysrhythmias are common and include bradycardia, atrioventricular conduction block or atrial arrhythmias. The mean age of death is 46 , with a high incidence of sudden death at $46 \%$ [1]. Unfortunately, pacemaker placement does not appear to reduce risk of sudden death, which is attributed to ventricular arrhythmias. 


\section{Sarcoglycanopathies}

The sarcoglycanopathies include four types of autosomal recessive Limb Girdle Muscular Dystrophies: LGMD 2C, $2 \mathrm{D}, 2 \mathrm{E}$ and $2 \mathrm{~F}$. These result in mutations in proteins of the sarcoglycan complex, part of the dystrophin-glycoprotein complex [1]. Patients typically present around age 8 with pelvic muscle and scapular weakness. While significant cardiac involvement in low in LGMD 2D, patients with LGMD 2E develop dilated cardiomyopathy and ventricular arrhythmias [1]. To assess for cardiac involvement, patients should be screened with an EKG and echocardiogram every 3-5 years [5].

\section{Fascioscapular Muscular Dystrophy}

Fascioscapular muscular dystrophy is an autosomal dominant disorder with a prevalence of 1 in 20,000 [2]. Progression is variable and clinically patients have facial, scapular and upper extremity weakness. Symptoms present between 16 and 20 years of age [1]. Fortunately, clinically significant cardiac disease is rare and when present includes arrhythmias such as supraventricular paroxysmal tachycardia.

\section{Congenital Muscular Dystrophy}

Congenital muscular dystrophy includes multiple inherited disorders which typically present within the first 6 months of life [1]. Clinical course is variable and cardiac involvement is disease specific. Patients may have cardiac involvement, with reduced left ventricular systolic function, however this is not typically clinically significant.

\section{Myotonic Dystrophy}

The myotonic dystrophies include two autosomal dominant disorders: myotonic dystrophy type 1 and type 2 . DM1 is the most common adult form of muscular dystrophy with a prevalence of 2.1-14.3 in 100,000 [1]. DM1 results from an expansion of the trinucleotide CTG in the 3' untranslated region of the myotonic dystrophy protein kinase (DMPK) gene. The disease demonstrates anticipation with expanded repeats in subsequent generations, producing more severe disease. The congenital form carries a high risk of death in the neonatal period and infants should have an echocardiogram at time of diagnosis $[1,2]$. The classic form presents between ages 20 and 40 years. Symptomatically, patients have myotonia and weakness of the facial, sternocleidomastoid and distal muscles. Patients also develop cataracts, gastrointestinal problems and cardiac involvement [1].

Cardiac involvement can occur by the second decade with conduction defects seen in $65 \%$ of adults. While the prevalence of congestive heart failure is low at 2-7\%, myotonic dystrophy patients have a significant risk of heart block and sudden death [1]. Sudden death was previously attributed to conduction blocks, however spontaneous ventricular tachycardias have been found to be a significant cause of death as well, even in patients with implantable pacemakers [1]. A previous comprehensive review of DM1 found that atrial flutter and fibrillation were the most common arrhythmias seen while ventricular arrhythmias were less common, but represented major management problems [1]. DM1 patients should be screened with a yearly EKG [1]. Holter monitoring should be performed in patients with EKG abnormalities as a means for detecting asymptomatic arrhythmias or conduction block [1]. Accordingly, myotonic dystrophy patients have a reduced life expectancy and death by cardiac causes is $20-30 \%$ [1]. The mean age of death is 53 years [2].

Myotonic dystrophy type 2 is similar to adult-onset DM1, but with a better clinical course. This condition results from an unstable expanded CCTG-repeat. Patients typically present later in life with proximal lower limb and finger flexor weakness. Conduction abnormalities are found in $20-36 \%$ of patients [1]. The risk of severe cardiac involvement is low; however patients should have an annual EKG.

\section{Treatment}

Although curative treatment is not yet available, quality of life and survival of muscular dystrophy patients have improved as a result of advances in medical management based on multi-disciplinary care [1].

Daily steroid therapy became the "gold standard" in DMD in 2004 [4]. Studies have showed that only 5\% of patients treated with deflazacort for $>3$ years had a significantly decreased EF, compared with $58 \%$ of untreated patients [6]. There was also a correlation between preserved cardiac function and pulmonary/skeletal muscle function. Interestingly, patients who had received steroids, but stopped taking them, showed normal cardiac function and no significant differences compared to those who had continued to take steroids. DMD patients treated with steroids prior 
to the onset of cardiac dysfunction show slower progression of heart disease [4]. The best type of steroid (prednisone vs. deflazacort), age of therapy initiation, duration of treatment, and dosing schedule still have to studied further.

The decreased cardiac function seen in cardiomyopathies stimulates the renin-angiotensin system and leads to the release of angiotensin II [7]. This leads to the stimulation of transforming growth factor- $\beta$, which promotes fibrosis. Angiotensin-converting enzyme inhibitors (ACEIs) modulate the production of angiotensin II and limit the amount of fibrosis/scarring in the myocardium. In DMD, early treatment with ACEIs delayed the onset and progression of left ventricular dysfunction and led to lower mortality in DMD [8]. ACEIs are becoming the primary therapy for cardiovascular disease in DMD and should be initiated at the first signs of decreased cardiac function. $\beta$-blockade should be used after ACEI is titrated to goal in advanced patients. The beneficial effect of $\beta$-blockade are well established in adults with cardiomyopathy, the role in children is less clear. Patients with DMD develop tachycardia as an early sign of cardiac dysfunction and blunting this response with $\beta$-adrenergic blockade has showed a reasonable response, though larger studies need to be done to determine the long-term efficacy [9]. Diuretics and digoxin have not shown a mortality benefit, but can be used as adjunctive treatments. For disordered automaticity, $\beta$-blocker therapy is used [3].

Cardiac transplantation is a viable option, particularly for the BMD population. Cardiac resynchronization therapy is a relatively new treatment option and should be considered in appropriate candidates in addition to implantable cardioverter-defibrillators [7]. Myotonic dystrophy patients with torsades de pointes and ventricular tachycardia may need implantable cardioverter-defibrillator placement [3].

Some new experimental approaches are noteworthy. Advances in the understanding of molecular genetics and the pathogenesis of muscular dystrophies have raised expectations of more effective therapy [1]. Poloxamer 188 is a nonionic triblock copolymer known to insert into artificial lipid monolayers and repair damaged biological membranes. In mouse models, administration during dobutamine infusion prevented the development of acute cardiac failure [10]. Continuous infusion into Golden Retriever muscular dystrophy dogs showed significantly decreased cardiac fibrosis and prevented ventricular dilation [11]. Based on these, P188 could become an important acute therapy in DMD during times of increased cardiac stress. Idebenone is a synthetic analog of coenzyme Q10 that has shown benefits in cardiac function in Friedreich ataxia [12]. In mice models simulating DMD, it was shown to prevent cardiac diastolic dysfunction, prevented dobutamine-induced acute cardiac failure, and decreased cardiac inflammation and fibrosis [4].

Gene therapy offers the promise of a cure by replacing the mutated dystrophin gene in all muscle tissues. Unfortunately, the dystrophin gene is too large to transfer by known vector systems, creating challenges. The basis of exon-skipping therapy is to use splice-switching oligonucleotides to bypass the mutated exam with a stop codon and continue to translate a smaller, truncated dystrophin protein. The goal would be to produce a BMD phenotype with a partially functional dystrophin protein expressed in muscle tissues [4]. A small open-label study found that weekly intravenous administration of the exon 51 skipping drug eteplirsen induced a dose-related increase in dystrophin production without drug-related adverse effects [13]. Another open-label study reported that systemic weekly subcutaneous administration of an antisense oligonucleotide (PRO051 or drisapersen) was associated with new dystrophin expression in 10 of 12 patients with DMD [14]. This led to an improvement in the six minute walk test in $8 / 12$ patients, but statistical significance was not seen at one year. Ataluren is an investigational orally administered drug being developed for the treatment of genetic defects caused by nonsense (stop) mutations. This approach could benefit the estimated 10 to 15 percent of patients with DMD/BMD who harbor nonsense (stop) mutations. The results of a phase 3 multicenter, double-blind, placebo-controlled trial of low-dose ataluren are awaited [15].

\section{Cardiac involvement in Myasthenia Gravis}

Myasthenia gravis is an acquired autoimmune disease caused by antibody attack on acetylcholine receptors in the postsynaptic membrane at the neuromuscular junction. It presents with fluctuating fatigable weakness of extraocular, bulbar, respiratory and limb muscles. Approximately 10\% of myasthenic cases are associated with thymoma. Other autoimmune diseases occur with increased frequency in myasthenic patients. Hypothyroidism is relatively common and can exascerbate myasthenic weakness.

Although the presenting symptoms of myasthenia gravis do not often involve the heart, associated cardiovascular dysfunction can be a source of morbity and mortality. The fundamental pathogenic agents in myasthenia, antibodies to skeletal muscle Ach receptors, do not bind to cardiac muscle [16]. However, other antibodies seen in myasthenic patients may react with myocardium. In addition, Ach receptor antibodies react with portions of the autonomic nervous 
system leading to cardiac arrhythmias or other disturbances of hemodynamics.

\section{Myocarditis}

Autopsy studies of myasthenic patients dating back to the early twentieth century have revealed focal inflammation and necrosis in myocardium [17]. This occurs more commonly but not exclusively in cases with thymoma. The severity of pathologic involvement varies within autopsy series and it is not clear that milder findings represent clinically significant heart disease [18]. At the other end of the spectrum, rampant myocarditis is considered the cause of death in the most severely affected cases.

The most dramatic and fully documented cases of myasthenia-related myocarditis have involved patients with thymoma. Pathology in such cases typically reveals giant multinucleated myocytes and histiocytes along with lymphocytic infiltrates in both skeletal and cardiac muscle. Treatment of this giant cell myocarditis is generally difficult; the disease is often fulminant and most reported cases have succumbed to heart failure or arrhythmia [19, 20].

The pathogenesis of myocarditis in myasthenic patients likely involves antistriational antibodies. The term antistriational includes antibodies to titin (a sarcolemmal protein), ryanodine receptors (within the sarcoplasmic reticulum) as well as Kv1.4 (a voltage gated potassium channel). In one series, over one third of myasthenic patients exhibiting antistriational antibodies developed myocarditis. Of these, $63 \%$ showed antibodies to titin, with antiryanodine and anti-Kv1.4 antibodies each found in 75\% [21]. Another series of 650 myasthenic patients reported that myocarditis was clinically suspected in about $12 \%$ of those with antibodies to Kv 1.4 but in none of those without [16].

\section{Takotsubo Cardiomyopathy}

A number of case reports have documented that Takotsubo cardiomyopathy can complicate the course of myasthenic crisis [22 - 25]. This transient ballooning of the left ventricle is also known as stress-induced cardiomyopathy or broken heart syndrome. It likely represents a myocardial reaction to catecholamine surges. Tokatsubo cardiomyopathy can occur in the setting of any critical illness and it is unclear whether myasthenic crisis poses particularly high risk. However, studies demonstrating exaggerated cardiac sensitivity to cathecholamines in myasthenia suggest a possible predisposition [26].

\section{Arrhythmia}

Retrospective series have demonstrated abnormalities of heart rate and rhythm in myasthenic patients [26, 27]. These may reflect complications of myocarditis (see above) or alternatively, disturbance of function within the autonomic nervous system. Both of these mechanisms appear to be more prevalent in myasthenic patients with thymoma. The dysautonomia accompanying thymoma can reflect circulating antibodies that bind specifically to neuronal Ach receptors in sympathetic and parasympathetic ganglia. Patients with these ganglionic Ach receptor antibodies can present with cardiac arrhythmia as part of a pan-dysautonomia with abnormalities of gastric motility, othostatic hypotension and sudomotor activity [28].

\section{Treatment}

Giant cell myocarditis is often poorly responsive to treatment and can be rapidly fatal despite intensive immunosuppressive regimens. Nevertheless, there are reported cases of survival with only minimal lasting cardiac and mysasthenic symtoms following treatment with high dose steroids, IV IG and placement of an indwelling pacemaker [16].

Tokatsubo cardiomyopathy generally has a good longterm prognosis. Supportive treatment includes positive inotropes and afterload reduction, at times requiring and aortic balloon pump. Hemodynamic considerations could limit the use of IVIG and plasmapheresis for concurrent myasthenic crisis but complications from other forms of immunomodulation have not been reported. The use of beta blockers may be particularly important in cases with ventricular outflow obstruction and their potential for worsening myasthenia would only be a secondary consideration.

Arrhythmia related to dysautonomia from antiganglionic Ach receptor antibodies will often improve with anticholinesterase or immunomodulatory treatment [28]. The use of cholinesterase inhibitors needs to be tempered by their potential to worsen bradyarrhythmia. 


\section{CONCLUSION}

Cardiac abnormalities can complicate the course of several muscular dystrophies. Progressive cardiomyopathy is the rule in Duchennes and Beckers musculardystrophies. In myotonic and Emery Dreifus muscular dystrophies, conduction abnormalities predominate and require regular surveillance. The risk of cardiac abnormalities varies among LimbGirdle muscular dystrophies, with laminopathy and sarcoglycanopathy respectively predisposing to heart block and cardiomyopathy. In myasthenia gravis, cardiovascular morbity occurs primarily in cases associated with thymoma.

\section{CONFLICT OF INTEREST}

The authors confirm that this article content has no conflict of interest.

\section{ACKNOWLEDGEMENTS}

Declared none.

\section{REFERENCES}

[1] Hermans MC, Pinto YM, Merkies IS, de Die-Smulders CE, Crijns HJ, Faber CG. Hereditary muscular dystrophies and the heart. Neuromuscul Disord 2010; 20(8): 479-92. [http://dx.doi.org/10.1016/j.nmd.2010.04.008] [PMID: 20627570]

[2] Beynon RP, Ray SG. Cardiac involvement in muscular dystrophies. QJM 2008; 101(5): 337-44. [http://dx.doi.org/10.1093/qjmed/hcm124] [PMID: 18238819]

[3] Allen HD, Thrush PT, Hoffman TM, Flanigan KM, Mendell JR. Cardiac management in neuromuscular diseases. Phys Med Rehabil Clin N Am 2012; 23(4): 855-68. [http://dx.doi.org/10.1016/j.pmr.2012.08.001] [PMID: 23137741]

[4] Spurney CF. Cardiomyopathy of Duchenne muscular dystrophy: current understanding and future directions. Muscle Nerve 2011; 44(1): 8-19. [http://dx.doi.org/10.1002/mus.22097] [PMID: 21674516]

[5] Hsu DT. Cardiac manifestations of neuromuscular disorders in children. Paediatr Respir Rev 2010; 11(1): 35-8. [http://dx.doi.org/10.1016/j.prrv.2009.10.004] [PMID: 20113990]

[6] Silversides CK, Webb GD, Harris VA, Biggar DW. Effects of deflazacort on left ventricular function in patients with Duchenne muscular dystrophy. Am J Cardiol 2003; 91(6): 769-72. [http://dx.doi.org/10.1016/S0002-9149(02)03429-X] [PMID: 12633823]

[7] Yilmaz A, Sechtem U. Cardiac involvement in muscular dystrophy: advances in diagnosis and therapy. Heart 2012; 98(5): 420-9. [http://dx.doi.org/10.1136/heartjnl-2011-300254] [PMID: 22311853]

[8] Duboc D, Meune C, Pierre B, et al. Perindopril preventive treatment on mortality in Duchenne muscular dystrophy: 10 years' follow-up. Am Heart J 2007; 154(3): 596-602. [http://dx.doi.org/10.1016/j.ahj.2007.05.014] [PMID: 17719312]

[9] Romfh A, McNally EM. Cardiac assessment in duchenne and becker muscular dystrophies. Curr Heart Fail Rep 2010; 7(4): 212-8. [http://dx.doi.org/10.1007/s11897-010-0028-2] [PMID: 20857240]

[10] Yasuda S, Townsend D, Michele DE, Favre EG, Day SM, Metzger JM. Dystrophic heart failure blocked by membrane sealant poloxamer. Nature 2005; 436(7053): 1025-9.

[http://dx.doi.org/10.1038/nature03844] [PMID: 16025101]

[11] Townsend D, Turner I, Yasuda S, et al. Chronic administration of membrane sealant prevents severe cardiac injury and ventricular dilatation in dystrophic dogs. J Clin Invest 2010; 120(4): 1140-50. [http://dx.doi.org/10.1172/JCI41329] [PMID: 20234088]

[12] Hausse AO, Aggoun Y, Bonnet D, et al. Idebenone and reduced cardiac hypertrophy in Friedreich's ataxia. Heart 2002; 87(4): 346-9. [http://dx.doi.org/10.1136/heart.87.4.346] [PMID: 11907009]

[13] Cirak S, Arechavala-Gomeza V, Guglieri M, et al. Exon skipping and dystrophin restoration in patients with Duchenne muscular dystrophy after systemic phosphorodiamidate morpholino oligomer treatment: an open-label, phase 2, dose-escalation study. Lancet 2011; 378(9791): 595-605.

[http://dx.doi.org/10.1016/S0140-6736(11)60756-3] [PMID: 21784508]

[14] Goemans NM, Tulinius M, van den Akker JT, et al. Systemic administration of PRO051 in Duchenne's muscular dystrophy. N Engl J Med $2011 ; 364(16): 1513-22$. [http://dx.doi.org/10.1056/NEJMoa1011367] [PMID: 21428760]

[15] Bushby K, Finkel R, Wong B, et al. Ataluren treatment of patients with nonsense mutation dystrophinopathy. Muscle Nerve 2014; 50(4): 477-87. [http://dx.doi.org/10.1002/mus.24332] [PMID: 25042182]

[16] Suzuki S, Baba A, Kaida K, et al. Cardiac involvements in myasthenia gravis associated with anti-Kv1.4 antibodies. Eur J Neurol 2014; 
21(2): 223-30.

[http://dx.doi.org/10.1111/ene.12234] [PMID: 23829303]

[17] Weigert C. Pathogisch-anatomischer Beitrag zur Erb’schen Krankheit (myasthenia gravis). Neurol Zentralbl 1943; $20: 597$.

[18] Hofstad H, Ohm OJ, Mørk SJ, Aarli JA. Heart disease in myasthenia gravis. Acta Neurol Scand 1984; 70(3): $176-84$. [http://dx.doi.org/10.1111/j.1600-0404.1984.tb00817.x] [PMID: 6507031]

[19] Sasaki H, Yano M, Kawano O, Hikosaka Y, Fujii Y. Thymoma associated with fatal myocarditis and polymyositis in a 58-year-old man following treatment with carboplatin and paclitaxel: A case report. Oncol Lett 2012; 3(2): 300-2. [PMID: 22740899]

[20] Kon T, Mori F, Tanji K, Miki Y, Kimura T, Wakabayashi K. Giant cell polymyositis and myocarditis associated with myasthenia gravis and thymoma. Neuropathology 2013; 33(3): 281-7.

[http://dx.doi.org/10.1111/j.1440-1789.2012.01345.x] [PMID: 22989101]

[21] Suzuki S, Utsugisawa K, Nagane Y, Suzuki N. Three types of striational antibodies in myasthenia gravis. Autoimmune Dis 2011. [http://dx.doi.org/10.4061/2011/740583]

[22] Bansal V, Kansal MM, Rowin J. Broken heart syndrome in myasthenia gravis. Muscle Nerve 2011; 44(6): $990-3$. [http://dx.doi.org/10.1002/mus.22220] [PMID: 22102473]

[23] Arai M, Ukigai H, Miyata H. A case of transient left ventricular ballooning ("Takotsubo"-shaped cardiomyopathy) developed during plasmapheresis for treatment of myasthenic crisis. Rinsho Shinkeigaku 2004; 44(3): 207-10. [PMID: 15233276]

[24] Mayor-Gomez S, Lacruz F, Ezpeleta D. Myasthenic crisis and Takotsubo syndrome: a non-chance relationship. Rev Neurol 2012; 55(12): 725-8.

[PMID: 23233140]

[25] Antevil JL, Carroll CG, Roberts PF, Johnston MG, Strange RG. Myasthenia gravis-an unexpected cause of respiratory failure and reversible left ventricular dysfunction after cardiac surgery. J Card Surg 2010; 25(6): 662-4. [http://dx.doi.org/10.1111/j.1540-8191.2010.01093.x] [PMID: 20796093]

[26] Shukla G, Gupta S, Goyal V, Singh S, Srivastava A, Behari M. Abnormal sympathetic hyper-reactivity in patients with myasthenia gravis: a prospective study. Clin Neurol Neurosurg 2013; 115(2): 179-86. [http://dx.doi.org/10.1016/j.clineuro.2012.05.013] [PMID: 22676958]

[27] Hofstad H, Ohm OJ, Mørk SJ, Aarli JA. Heart disease in myasthenia gravis. Acta Neurol Scand 1984; 70(3): $176-84$. [http://dx.doi.org/10.1111/j.1600-0404.1984.tb00817.x] [PMID: 6507031]

[28] Vernino S, Cheshire WP, Lennon VA. Myasthenia gravis with autoimmune autonomic neuropathy. Auton Neurosci 2001 ; 88(3): 187-92. [http://dx.doi.org/10.1016/S1566-0702(01)00239-9] [PMID: 11474561]

(C) Hickey et al.; Licensee Bentham Open.

This is an open access article licensed under the terms of the Creative Commons Attribution-Non-Commercial 4.0 International Public License (CC BY-NC 4.0) (https://creativecommons.org/licenses/by-nc/4.0/legalcode), which permits unrestricted, non-commercial use, distribution and reproduction in any medium, provided the work is properly cited. 